JOURNAL OF

SYMPLECTIC GEOMETRY

Volume 11, Number 3, 449-474, 2013

\title{
ACTION-INDEX RELATIONS FOR PERFECT HAMILTONIAN DIFFEOMORPHISMS
}

\author{
Mike Chance, Viktor Ginzburg, and Başak Gürel
}

We show that the actions and indexes of fixed points of a Hamiltonian diffeomorphism with finitely many periodic points must satisfy certain relations, provided that the quantum cohomology of the ambient manifold meets an algebraic requirement satisfied for projective spaces, Grassmannians and many other manifolds. We also refine a previous result on the Conley conjecture for negative monotone symplectic manifolds, due to the second and third authors, and show that a Hamiltonian diffeomorphism of such a manifold must have simple periodic orbits of arbitrarily large period whenever its fixed points are isolated.

\section{Contents}

1. Introduction and main results $\quad 450$

1.1. Introduction $\quad 450$

1.2. Action-index relations 450

1.3. Conley conjecture for negative monotone symplectic manifolds

2. Preliminaries

2.1. Conventions and notation

2.2. Floer and quantum (co)homology 


\section{Introduction and main results}

1.1. Introduction. The central theme of this paper is a rigidity feature of Hamiltonian diffeomorphisms with finitely many periodic points. To be more specific, we prove that there are certain relations between the actions and indexes of fixed points of such a diffeomorphism, provided that the quantum cohomology of the ambient manifold meets an algebraic requirement, which is fully satisfied for projective spaces, Grassmannians, and to a degree in all known monotone examples. We also refine our previous result on the Conley conjecture for negative monotone symplectic manifolds (see [GG3]) and show that a Hamiltonian diffeomorphism of such a manifold must have simple periodic orbits of arbitrarily large period whenever its fixed points are isolated.

The fact that there are some relations between the mean indexes and/or actions of fixed points of a Hamiltonian system with finitely many periodic orbits is not new. For indexes of a Reeb flow on the standard sphere it goes back to $[\mathbf{E k}, \mathbf{E H}, \mathbf{V i 1}]$ and it has been further explored and generalized since then; see, e.g., $[\mathbf{E s}, \mathbf{G K}]$. On the other hand, the observation that the mean indexes, or indexes and actions, of fixed points of a Hamiltonian diffeomorphism (of, say, $\mathbb{C P}^{n}$ ) with finitely many periodic orbits must meet certain algebraic conditions is relatively recent; see $[\mathbf{G K}]$ for resonance relations for indexes and [GG1] for action-index relations. (Interestingly, no analogue of action-index relations in the contact case appears to have been established so far.) The main focus of this paper is a treatment of the action-index relations in a more systematic way that connects them with the algebraic properties of the quantum homology of the ambient manifold.

The paper is organized as follows. Below, in Sections 1.2 and 1.3, we state the main results of the paper - the action-index relations and a refinement of the Conley conjecture for negative monotone manifolds. In Section 2, we set our conventions and notation and discuss some standard (and not entirely standard) notions and results from symplectic topology, needed for the proof of the main theorems. These include the mean index, the filtered and local Floer homology, the action selector and the action selector carrier, and their properties. Then, in Section 3, we prove Theorem 1.1 establishing the existence of action-index relations. Theorem 1.8 (a refinement of the Conley conjecture for negative monotone manifolds) is proved in Section 4 .

1.2. Action-index relations. Throughout this paper, $(M, \omega)$ will stand for a monotone or negative monotone closed symplectic manifold of dimension $2 n$ with monotonicity constant $\lambda$ and minimal Chern number $N$. (We 
refer the reader to Section 2 for the definitions and a detailed discussion of the notions used in this section.) Here, we only note that we may assume from now on that $0<N<\infty$, i.e., $\left.c_{1}(M)\right|_{\pi_{2}(M)} \neq 0$ in $\mathbb{R}$. For otherwise the conditions of Theorem 1.1 below are never satisfied and Theorem 1.8 is known to hold. (This is a consequence of the Conley conjecture for symplectic manifolds with $\left.c_{1}(M)\right|_{\pi_{2}(M)}=0$, proved in $[\mathbf{G G 1 , H e ]}$; see also $[\mathbf{G i}, \mathbf{H i}]$.) We will also usually require that $\lambda \neq 0$.

Let $\varphi_{\mathrm{H}}$ be a Hamiltonian diffeomorphism of $M$ generated by a oneperiodic in time Hamiltonian $H: S^{1} \times M \rightarrow \mathbb{R}$ with $S^{1}=\mathbb{R} / \mathbb{Z}$. We denote by $\mathcal{P}$ the collection of contractible one-periodic orbits of the time-dependent flow $\varphi_{\mathrm{H}}^{t}$ and by $\overline{\mathcal{P}}$ the collection of capped one-periodic orbits. Clearly, $\mathcal{P}$ can be viewed as a subset of the fixed point set of $\varphi_{\mathrm{H}}$. For $x \in \mathcal{P}$, the augmented action of $H$ on $x$ is defined by

$$
\tilde{\mathcal{A}}_{\mathrm{H}}(x)=\mathcal{A}_{\mathrm{H}}(\bar{x})-\frac{\lambda}{2} \Delta_{\mathrm{H}}(\bar{x}),
$$

where $\mathcal{A}_{\mathrm{H}}(\bar{x})$ and $\Delta_{\mathrm{H}}(\bar{x})$ stand for the action and, respectively, the mean index of a capped orbit $\bar{x}$; see Section 2.1. Since $\mathcal{A}_{\mathrm{H}}(\bar{x})$ and $\Delta_{H}(\bar{x})$ change in the same way under recapping, up to the factor $\lambda / 2$, the augmented action is well defined, i.e., independent of the capping.

We denote the quantum cohomology of $M$ over a ground field $\mathbb{F}$ by $\mathrm{HQ}^{*}(M ; \mathbb{F})$ or simply by $\mathrm{HQ}^{*}(M)$ when the role of $\mathbb{F}$ is inessential. The quantum product of elements $u$ and $v$ in $\mathrm{HQ}^{*}(M)$ will be denoted by $u * v$ and the degree of $u$ by $|u|$. Recall that the quantum cohomology is a module over the Novikov ring $\Lambda$. The version of $\Lambda$ we will utilize here is just the Laurent polynomial ring $\mathbb{F}\left[q, q^{-1}\right]$, where $|q|=2 N$. We refer the reader to Section 2.2.2 for more details on quantum (co)homology and further references.

Below we will use the ceiling and floor functions denoted by $\lceil\cdot\rceil$ and $\lfloor\cdot\rfloor$. Recall that $\lceil a\rceil$ stands for the least integer greater than or equal to $a$ and $\lfloor a\rfloor$ is the greatest integer smaller than or equal to $a$.

The main result of the paper is the following.

Theorem 1.1 (Action-index relations). Let $\varphi_{\mathrm{H}}$ be a Hamiltonian diffeomorphism with finitely many periodic orbits of a closed monotone symplectic manifold $M$ with $\lambda \neq 0$ and $0<N<\infty$.

(i) Assume that

$$
u_{0} * u_{1} * \cdots * u_{\ell}=q^{\nu} u_{0} \text { in } \mathrm{HQ}^{*}(M),
$$

where $\nu>0, u_{0} \neq 0$, the classes $u_{1}, \ldots, u_{\ell}$ have positive degree, and

$$
\left|u_{1}\right|+\cdots+\left|u_{\ell-1}\right|<2 N \text {. }
$$

Assume furthermore, unless $\nu=1$, that $\varphi_{\mathrm{H}}$ is non-degenerate. Then there exist $\ell$ distinct contractible one-periodic orbits $x_{0}, \ldots, x_{\ell-1}$ of 
$\varphi_{\mathrm{H}}^{t}$ such that

(ii) Alternatively, assume that there exists $u \in \mathrm{HQ}^{*>0}(M)$ such that

$$
u^{d} \neq 0
$$

for some sufficiently large $d$ (e.g., we can take $d=\lceil 2 N|\mathcal{P}| /|u|\rceil+1$ ) and that $\varphi_{\mathrm{H}}$ is non-degenerate. Then the assertion of (i) holds for $\ell=\lfloor 2 N /|u|\rfloor$.

We will call (1.1) a product decomposition of $u_{0}$. It is essential that the degree bound (1.2) involves neither the first term $u_{0}$ of the product decomposition nor the last one $u_{\ell}$. In particular, both $u_{0}$ and $u_{\ell}$ can have large degree not necessarily bounded by $2 N$. (However, clearly, $\left|u_{1}\right|+\cdots+\left|u_{\ell}\right|=2 N \nu$ and (1.2) is automatically satisfied when $\nu=1$.) It is also worth pointing out that in all known examples, which are in fact listed below, of Hamiltonian diffeomorphisms $\varphi_{\mathrm{H}}$ with finitely many periodic points, $\varphi_{\mathrm{H}}$ is non-degenerate and every periodic point of $\varphi_{\mathrm{H}}$ is a fixed point, i.e., $\varphi_{\mathrm{H}}$ is perfect in the terminology of $[\mathbf{G K}]$ - hence the title of the paper.

The theorem cannot produce more than $\ell=2 N$ fixed points with equal augmented action. (In fact, $\ell \leq N$ when $\operatorname{char}(\mathbb{F}) \neq 2$, since elements of odd degree do not contribute to product decompositions. Indeed, assume that, e.g., $\left|u_{1}\right|$ is odd. Then $u_{1}^{2}=0$ and, multiplying (1.1) by $u_{1} * \cdots * u_{\ell}$, we obtain that $0=q^{2 \nu} u_{0}$, which is impossible. The authors are grateful to the referee for this remark.) A difficulty here lies in showing that the points are distinct and is similar to the difficulty arising in establishing the degenerate case of the Arnold conjecture. However, as we will see, in all known examples all fixed points have equal augmented actions.

Let us now examine examples of Hamiltonian diffeomorphisms with finitely many periodic points.

Example 1.2. The simplest Hamiltonian diffeomorphism with finitely many periodic orbits is an irrational rotation of $S^{2}$. More generally, let $\varphi_{\mathrm{H}}$ be the Hamiltonian diffeomorphism of $\mathbb{C P}^{n}$, generated by a quadratic Hamiltonian $H(z)=\pi\left(\lambda_{0}\left|z_{0}\right|^{2}+\cdots+\lambda_{n}\left|z_{n}\right|^{2}\right)$, where the coefficients $\lambda_{0}, \ldots, \lambda_{n}$ are all distinct. (Here, we have identified $\mathbb{C P}^{n}$ with the quotient of the unit sphere in $\mathbb{C}^{n+1}$ and hence $\sum\left|z_{i}\right|^{2}=1$.) Now, (1.1) takes the form $u^{n+1}=q$, where $u$ is the first Chern class of the canonical line bundle, and $N=n+1=\ell$; see, e.g., [MS, Section 11.3]. The Hamiltonian diffeomorphism $\varphi_{\mathrm{H}}$ is perfect and has exactly $\ell$ fixed points (the coordinate axes). Their augmented actions are equal to $\pi \sum \lambda_{i} /(n+1)$. In this connection let us point out that there is a coefficient error in [GK, Example 1.2]. With our conventions (see Section 2.1), $\Delta\left(x_{j}\right)=2\left(-\sum \lambda_{i}+(n+1) \lambda_{j}\right)$, where $x_{j}$ is equipped with the trivial capping. 
This example fits in a much more general framework:

Example 1.3. Suppose that $(M, \omega)$ admits a Hamiltonian action of a torus $G$ with isolated fixed points; see, e.g., $[\mathbf{G G K}]$ for the definition and further details. A generic element of $G$ gives rise to a perfect Hamiltonian diffeomorphism $\varphi$ of $(M, \omega)$ whose fixed points are exactly the fixed points of the torus action. One can show that in this case all fixed points have the same augmented action, i.e., (1.3) holds for the entire collection of fixed points.

One way to see that this is true is as follows. Without loss of generality, we may assume that $\lambda=1$. Let $\vec{H}$ be the moment map of the action. Then the equivariant two-form $\omega^{G}=\omega+\vec{H}$ can be thought as simultaneously representing the equivariant Chern class $c_{1}^{G}(T M)$ and an equivariant extension $[\omega]^{G}$ of the symplectic form class. Since the fixed points of the action are isolated, the localization of the latter class is simply the sum of the moment map values $\vec{H}(x)$ for $x \in M^{G}$. On the other hand, it is not hard to see that for $c_{1}^{G}(T M)$ and each $x$ this is also the sum of the weights of the representation of $G$ on $T_{x} M$. Expressing $H(x)$ as a linear combination of the components of $\vec{H}(x)$ and $\Delta(x) / 2$ as a linear combination of the components of the sum of the weights, we conclude that $H(x)=\Delta(x) / 2$. (The authors are grateful to Yael Karshon for this argument.)

Examples of symplectic manifolds that admit such torus actions include a majority of coadjoint orbits of compact Lie groups, e.g., complex Grassmannians $G(k, N)$. One can also construct new examples from a given one by equivariantly blowing-up the symplectic manifold at fixed points. The resulting symplectic manifold always inherits a Hamiltonian torus action and, in many instances, this action also has isolated fixed points.

Example 1.4. Hamiltonian diffeomorphisms with finitely many periodic orbits need not be associated with Hamiltonian torus actions as in Example 1.3. For instance, there exists a Hamiltonian perturbation $\varphi$ of an irrational rotation of $S^{2}$ with exactly three ergodic invariant measures: the Lebesgue measure and the two measures corresponding to the fixed points of $\varphi ;$ see $[\mathbf{F H}, \mathbf{F K}]$ and also, for relevant results, $[\mathbf{A K}]$. Taking direct products of such diffeomorphisms we obtain examples in higher dimensions. It is easy to see from the construction of $\varphi$ that in this case all fixed points have again the same augmented action. (This also follows from Theorem 1.1 since $\varphi$ has exactly two fixed points.)

To the best of our knowledge, Examples 1.3 and 1.4 and their products exhaust the list of known Hamiltonian diffeomorphisms with finitely many periodic orbits.

Product decompositions with $\ell \geq 2$ depending on the manifold in question exist for Grassmannians $G(k, N)$ and their monotone products, as is easy to see from the description of the quantum product on $G(k, N)$; see, 
e.g., [ST, MS, Ta] for further references and details. Moreover, once a product decomposition exists for $M$ it also exists, with the same $\ell$, for any monotone product of the form $M \times P$. (This follows from the quantum Künneth formula; $[\mathbf{K a}]$.) Here, for instance, $P$ can be symplectically aspherical although in this case no products $M \times P$ admitting perfect Hamiltonian diffeomorphisms are known. However, "long" product decompositions are difficult find. For instance, we have not been able to show that in general $G(k, N)$ admits a product decomposition of length $N$ (the minimal Chern number) and this is where Case (ii) of the theorem becomes useful.

Corollary 1.5. Let $M$ be $G(k, N)$ or, more generally, a monotone product $G(k, N) \times P$, and let $\varphi_{\mathrm{H}}$ be a Hamiltonian diffeomorphism of $M$ with finitely many fixed points. Then there exist $\ell=N$ distinct fixed points $x_{0}, \ldots, x_{\ell-1}$ of $\varphi_{\mathrm{H}}$ satisfying (1.3).

Proof. Let $u$ be the first Chern class of the canonical vector bundle over $G(k, N)$. Then $u^{d} \neq 0$, for any $d>0$, in $\operatorname{HQ}^{*}(G(k, N) ; \mathbb{Q})$. This is an immediate consequence of quantum Schubert calculus and, more precisely, of the quantum Pieri formula; see $[\mathbf{B e}]$ and also, e.g., [Ta]. Applying the quantum Künneth formula, we see that $u^{d} \neq 0$ for $M=G(k, N) \times P$; see $[\mathbf{K a}]$ and also [MS, Exercise 11.1.15]. Now the corollary follows from Case (ii) of Theorem 1.1.

Remark 1.6. The hypotheses of Cases (i) and (ii) of the theorem are in fact related. For instance, when the ground field $\mathbb{F}$ is finite, the existence of an element $u$ with $u^{d} \neq 0$ for all $d \geq 0$ implies, by the pigeonhole principle, a product decomposition of length $\ell=\lfloor 2 N /|u|\rfloor$. This argument however cannot be used to find a product decomposition for $G(k, N)$ of length $N$ : $u^{3}=0$ in $\operatorname{HQ}^{*}\left(G(2,4) ; \mathbb{Z}_{2}\right)$ and it is absolutely essential for the proof of Corollary 1.5 that $\mathbb{F}$ has zero characteristic.

Remark 1.7. Theorem 1.1 generalizes Corollary 1.11 and Theorem 1.12 in $[\mathbf{G G 1}$, where the augmented action was originally defined. However, a similar notion (the action-index) was considered in $[\mathbf{P o}]$ in a different context for a loop of Hamiltonian diffeomorphisms. Furthermore, replacing the mean index in the definition of the augmented action by some other version of the Conley-Zehnder index (as in, e.g., [Sa, Section 1.6]), we still obtain a well-defined, i.e., independent of capping, invariant. Theorem 1.1 would not hold for such an invariant. What sets apart the augmented action, defined as above using the mean index, is that it is homogeneous with respect to iterations of $\varphi_{\mathrm{H}}$.

1.3. Conley conjecture for negative monotone symplectic manifolds. Our proof of Theorem 1.1 builds on the proof of the Conley conjecture for negative monotone symplectic manifolds in [GG3]. In turn, the 
proof of Theorem 1.1 lends itself readily to the following refinement of the main result of [GG3].

Theorem 1.8. Let $\varphi_{\mathrm{H}}$ be a Hamiltonian diffeomorphism of a closed, negative monotone symplectic manifold. Assume that $\varphi_{\mathrm{H}}$ has finitely many fixed points. Then $\varphi_{\mathrm{H}}$ has simple periodic orbits of arbitrarily large period.

Negative monotone manifolds exist in abundance. Among them are, for instance, the hypersurfaces $z_{0}^{k}+\cdots+z_{n}^{k}=0$ in $\mathbb{C P}^{n}$ with $N=k-(n+1)>0$; see, e.g., [MS, pp. 429-430] and also [LM, p. 88] for further examples.

Remark 1.9. The new point here, as compared to [GG3], is the existence of simple periodic orbits with arbitrarily large period rather than just the existence of infinitely many periodic orbits.

The proof of Theorem 1.8 utilizes Hamiltonian Floer theory. Hence, unless $M$ is required to be weakly monotone, the argument ultimately, although not explicitly, relies on the machinery of multi-valued perturbations and virtual cycles; see Remark 2.1 for further discussion.

\section{Preliminaries}

The goal of this section is to set notation and conventions, following mainly [GG1, GG3], and to give a brief review of Floer homology and several other notions used in the paper focusing on the case where $M$ is monotone or negative monotone with $\left.c_{1}(M)\right|_{\pi_{2}(M)} \neq 0$.

2.1. Conventions and notation. Let $\left(M^{2 n}, \omega\right)$ be a closed symplectic manifold. Throughout the paper, we will assume $M$ to be monotone as in Theorem 1.1 or negative monotone as in Theorem 1.8, i.e., $\left.[\omega]\right|_{\pi_{2}(M)}=$ $\left.\lambda c_{1}(M)\right|_{\pi_{2}(M)}$ for some $\lambda \geq 0$ in the former case or $\lambda<0$ in the latter. In particular, $M$ is rational, i.e., the group $\left\langle[\omega], \pi_{2}(M)\right\rangle \subset \mathbb{R}$ formed by the integrals of $\omega$ over the spheres in $M$ is discrete. The positive generator $\lambda_{0}$ of this group is called the rationality constant. In the monotone case we will usually require that $\lambda \neq 0$.

All Hamiltonians $H$ on $M$ considered in this paper are assumed to be $k$-periodic in time, i.e., $H: S_{k}^{1} \times M \rightarrow \mathbb{R}$, where $S_{k}^{1}=\mathbb{R} / k \mathbb{Z}$, and the period $k$ is always a positive integer. When the period is not specified, it is equal to one, which is the default period in this paper. We set $H_{t}=H(t, \cdot)$ for $t \in S^{1}=\mathbb{R} / \mathbb{Z}$. The Hamiltonian vector field $X_{\mathrm{H}}$ of $H$ is defined by $i_{X_{\mathrm{H}}} \omega=-d H$. The (time-dependent) flow of $X_{\mathrm{H}}$ will be denoted by $\varphi_{\mathrm{H}}^{t}$ and its time-one map by $\varphi_{\mathrm{H}}$. Such time-one maps are referred to as Hamiltonian diffeomorphisms. A one-periodic Hamiltonian $H$ can always be treated as $k$ periodic. In this case, we will use the notation $H^{\sharp k}$ and, abusing terminology, call $H^{\sharp k}$ the $k$ th iteration of $H$. 
Let $K$ and $H$ be one-periodic Hamiltonians such that $K_{1}=H_{0}$ and $H_{1}=K_{0}$. We denote by $K \sharp H$ the two-periodic Hamiltonian equal to $K_{t}$ for $t \in[0,1]$ and $H_{t-1}$ for $t \in[1,2]$. Thus, $H^{\sharp k}=H \sharp \cdots \sharp H$ (k times).

Let $x: S_{k}^{1} \rightarrow W$ be a contractible loop. A capping of $x$ is a map $u: D^{2} \rightarrow M$ such that $\left.u\right|_{S_{k}^{1}}=x$. Two cappings $u$ and $v$ of $x$ are considered to be equivalent if the integrals of $\omega$ and $c_{1}(M)$ over the sphere obtained by attaching $u$ to $v$ are equal to zero. A capped closed curve $\bar{x}$ is, by definition, a closed curve $x$ equipped with an equivalence class of cappings. In what follows, the presence of capping is always indicated by the bar.

The action of a one-periodic Hamiltonian $H$ on a capped closed curve $\bar{x}=(x, u)$ is defined by

$$
\mathcal{A}_{\mathrm{H}}(\bar{x})=-\int_{u} \omega+\int_{S^{1}} H_{t}(x(t)) d t .
$$

The space of capped closed curves is a covering space of the space of contractible loops and the critical points of $\mathcal{A}_{\mathrm{H}}$ on the covering space are exactly capped one-periodic orbits of $X_{\mathrm{H}}$. The action spectrum $\mathcal{S}(H)$ of $H$ is the set of critical values of $\mathcal{A}_{\mathrm{H}}$. This is a zero measure set; see, e.g., [HZ, Sc]. When $M$ is rational, $\mathcal{S}(H)$ is closed, and hence nowhere dense. Otherwise, $\mathcal{S}(H)$ is dense in $\mathbb{R}$. These definitions extend to $k$-periodic orbits and Hamiltonians in an obvious way. Clearly, the action functional is homogeneous with respect to iteration:

$$
\mathcal{A}_{H \sharp k}\left(\bar{x}^{k}\right)=k \mathcal{A}_{\mathrm{H}}(\bar{x}) .
$$

Here $\bar{x}^{k}$ stands for the $k$ th iteration of the capped orbit $\bar{x}$.

The results of this paper concern only contractible periodic orbits and throughout the paper a periodic orbit is always assumed to be contractible, even if this is not explicitly stated.

A periodic orbit $x$ of $H$ is said to be non-degenerate if the linearized return $\operatorname{map} d \varphi_{\mathrm{H}}: T_{x(0)} W \rightarrow T_{x(0)} W$ has no eigenvalues equal to one. Following $[\mathbf{S Z}]$, we call $x$ weakly non-degenerate if at least one of the eigenvalues is different from one. A Hamiltonian is non-degenerate if all its one-periodic orbits are non-degenerate.

Let $\bar{x}$ be a non-degenerate (capped) periodic orbit. The Conley-Zehnder index $\mu_{\mathrm{CZ}}(\bar{x}) \in \mathbb{Z}$ is defined, up to a sign, as in $[\mathbf{S a}, \mathbf{S Z}]$. (Sometimes, we will also use the notation $\mu_{\mathrm{CZ}}(H, \bar{x})$.) More specifically, in this paper, the Conley-Zehnder index is the negative of that in [Sa]. In other words, we normalize $\mu_{\mathrm{CZ}}$ so that $\mu_{\mathrm{CZ}}(\bar{x})=n$ when $x$ is a non-degenerate maximum (with trivial capping) of an autonomous Hamiltonian with small Hessian. The mean index $\Delta_{\mathrm{H}}(\bar{x}) \in \mathbb{R}$ measures, roughly speaking, the total angle swept by certain eigenvalues with absolute value one of the linearized flow $d \varphi_{\mathrm{H}}^{t}$ along $x$ with respect to the trivialization associated with the capping; see $[\mathbf{L o}, \mathbf{S Z}]$. The mean index is defined regardless of whether $x$ is degenerate or not and $\Delta_{\mathrm{H}}(\bar{x})$ depends continuously on $H$ and $\bar{x}$ in the obvious sense. 
When $x$ is non-degenerate or just weakly non-degenerate, we have

$$
0<\left|\Delta_{\mathrm{H}}(\bar{x})-\mu_{\mathrm{CZ}}(H, \bar{x})\right|<n .
$$

Furthermore, the mean index is homogeneous with respect to iteration:

$$
\Delta_{H \sharp k}\left(\bar{x}^{k}\right)=k \Delta_{\mathrm{H}}(\bar{x}) .
$$

As a consequence, the augmented action is also homogeneous.

2.2. Floer and quantum (co)homology. Although the hypotheses of Theorem 1.1 are stated in terms of quantum cohomology, we find working with homology more intuitive in the context of Ljusternik-Schnirelman theory, which the proof of the theorem relies on. Hence, here we focus mainly on the definitions of quantum and Floer homology and just briefly indicate the modifications needed in the case of cohomology. The assumptions of Theorem 1.1 are reformulated via homology at the beginning of Section 3 .

2.2.1. Floer homology. In this subsection, we very briefly recall, mainly to set notation, the construction of the filtered Floer homology. We refer the reader to, e.g., $[\mathbf{H S}, \mathbf{M S}, \mathbf{S a}, \mathbf{S Z}]$ and also $[\mathbf{F O}, \mathbf{L T}]$ for detailed accounts and additional references.

Fix a ground field $\mathbb{F}$. Let $H$ be a non-degenerate Hamiltonian on $M$. Denote by $\mathrm{CF}_{k}^{(-\infty, b)}(H)$, where $b \in(-\infty, \infty]$ is not in $\mathcal{S}(H)$, the vector space of finite formal sums

$$
\sigma=\sum_{\bar{x} \in \overline{\mathcal{P}}(H)} \sigma_{\bar{x}} \bar{x}
$$

Here $\sigma_{\bar{x}} \in \mathbb{F}$ and $\mu_{\mathrm{CZ}}(\bar{x})+n=k$ and $\mathcal{A}_{\mathrm{H}}(\bar{x})<b$. (Since we focus on the case where $M$ is monotone with $\lambda \neq 0$ or negative monotone and $\left.c_{1}(M)\right|_{\pi_{2}(M)} \neq 0$ there is no need to consider semi-infinite sums.) We say that $\bar{x}$ enters the chain $\sigma$ when $\sigma_{\bar{x}} \neq 0$. The graded $\mathbb{F}$-vector space $\mathrm{CF}_{*}^{(-\infty, b)}(H)$ is endowed with the Floer differential counting the anti-gradient trajectories of the action functional; see, e.g., $[\mathbf{H S}, \mathbf{M S}, \mathbf{O n}, \mathbf{S a}]$ and also $[\mathbf{F O}, \mathbf{L T}]$. Thus, we obtain a filtration of the total Floer complex $\mathrm{CF}_{*}(H):=\mathrm{CF}_{*}^{(-\infty, \infty)}(H)$. Furthermore, we set $\mathrm{CF}_{*}^{(a, b)}(H):=\mathrm{CF}_{*}^{(-\infty, b)}(H) / \mathrm{CF}_{*}^{(-\infty, a)}(H)$, where $-\infty \leq a<b \leq \infty$ are not in $\mathcal{S}(H)$. The resulting homology, the filtered Floer homology of $H$, is denoted by $\operatorname{HF}_{*}^{(a, b)}(H)$ and by $\operatorname{HF}_{*}(H)$ when $(a, b)=(-\infty, \infty)$. Note that every $\mathbb{F}$-vector space $\mathrm{CF}_{k}(H)$ is finitedimensional since $M$ is negative monotone or monotone with $\lambda \neq 0$ and $\left.c_{1}(M)\right|_{\pi_{2}(M)} \neq 0$.)

The total Floer complex and homology are modules over the Novikov ring $\Lambda$, which in our case is simply a ring of Laurent polynomials. To be more specific, let $\omega(A)$ and $\left\langle c_{1}(M), A\right\rangle$ denote the integrals of $\omega$ and, respectively, 
$c_{1}(M)$ over a cycle $A$. Set

$$
I_{\omega}(A)=-\omega(A) \text { and } I_{c_{1}}(A)=-2\left\langle c_{1}(M), A\right\rangle,
$$

where $A \in \pi_{2}(M)$. Since $M$ is monotone or negative monotone,

$$
I_{\omega}=\frac{\lambda}{2} I_{c_{1}}
$$

and, in particular, $I_{\omega}(A)$ and $I_{c_{1}}(A)$ have opposite signs when $M$ is negative monotone. Let $\Gamma=\pi_{2}(M) / \operatorname{ker} I_{c_{1}}$. Thus, $\Gamma$ is the quotient of $\pi_{2}(M)$ by the equivalence relation where two spheres $A$ and $A^{\prime}$ are considered to be equivalent if $\omega(A)=\omega\left(A^{\prime}\right)$ (and hence $\left\langle c_{1}(M), A\right\rangle=\left\langle c_{1}(M), A^{\prime}\right\rangle$ ). The homomorphisms $I_{\omega}$ and $I_{c_{1}}$ descend from $\pi_{2}(M)$ to $\Gamma$.

The group $\Gamma$ acts on $\mathrm{CF}_{*}(H)$ and on $\mathrm{HF}_{*}(H)$ via recapping: an element $A \in \Gamma$ acts on a capped one-periodic orbit $\bar{x}$ of $H$ by attaching the sphere $A$ to the original capping. We denote the resulting capped orbit by $\bar{x} \# A$. Then,

$$
\mu_{\mathrm{CZ}}(\bar{x} \# A)=\mu_{\mathrm{CZ}}(\bar{x})+I_{c_{1}}(A) \quad \text { and } \quad \mathcal{A}_{\mathrm{H}}(\bar{x} \# A)=\mathcal{A}_{\mathrm{H}}(\bar{x})+I_{\omega}(A) .
$$

In a similar vein, we also have

$$
\Delta_{\mathrm{H}}(\bar{x} \# A)=\Delta_{\mathrm{H}}(\bar{x})+I_{c_{1}}(A),
$$

regardless of whether $x$ is non-degenerate or not.

The Novikov ring $\Lambda$ we consider here is the group algebra $\mathbb{F}[\Gamma]$ of $\Gamma$ over $\mathbb{F}$. Namely, $\Lambda$ comprises finite formal linear combinations $\sum \alpha_{A} e^{A}$, where $\alpha_{A} \in \mathbb{F}$ and $A \in \Gamma$. Clearly, $\Lambda$ is graded by setting $\left|e^{A}\right|=I_{c_{1}}(A)$ for $A \in \Gamma$. The action of $\Gamma$ turns $\mathrm{CF}_{*}(H)$ and $\mathrm{HF}_{*}(H)$ into $\Lambda$-modules.

Since $M$ is monotone or negative monotone and $\left.c_{1}(M)\right|_{\pi_{2}(M)} \neq 0$, we have $\Gamma \simeq \mathbb{Z}$. Denote by $A$ the generator of $\Gamma$ with $I_{c_{1}}(A)=-2 N$ and set $q=e^{A} \in \Lambda$. Then $|q|=-2 N$ and $\Lambda$ is the ring of Laurent polynomials $\mathbb{F}\left[q^{-1}, q\right]$.

The definition of Floer homology extends to all, not necessarily nondegenerate, Hamiltonians by continuity. Let $H$ be an arbitrary (one-periodic in time) Hamiltonian on $M$ and let the end points $a$ and $b$ of the action interval be outside $\mathcal{S}(H)$. We set

$$
\mathrm{HF}_{*}^{(a, b)}(H)=\operatorname{HF}_{*}^{(a, b)}(\tilde{H}),
$$

where $\tilde{H}$ is a non-degenerate, small perturbation of $H$. It is well known that the right hand side is independent of $\tilde{H}$ as long as the latter is sufficiently close to $H$. Working with filtered Floer homology, we will always assume that the end points of the action interval are not in the action spectrum. (At this point the background assumption that $M$ is rational becomes essential; see $[\mathbf{H e}]$ for the irrational case and also [GG1, Remark 2.3].) 
The total Floer homology is independent of the Hamiltonian and isomorphic to the homology of $M$. More precisely, we have

$$
\mathrm{HF}_{*}(H) \cong \mathrm{H}_{*}(M ; \mathbb{F}) \otimes \Lambda
$$

as graded $\Lambda$-modules.

Remark 2.1. We conclude this discussion by recalling that in order for the Floer differential to be defined certain regularity conditions must be satisfied generically. To ensure this, we have to either require $M$ to be weakly monotone (see [HS, MS, On, Sa]) or utilize the machinery of virtual cycles (see $[\mathbf{F O}, \mathbf{F O O O}, \mathbf{L T}]$ or, for the polyfold approach, $[\mathbf{H W Z 1 , H W Z 2}]$ and references therein). In the latter case the ground field $\mathbb{F}$ is required to have zero characteristic. Here, we are primarily interested in monotone manifolds, which are of course weakly monotone, and negative monotone manifolds. The latter are weakly monotone if and only if $N \geq n-2$.

2.2.2. Quantum (co)homology. The total Floer homology $\mathrm{HF}_{*}(H)$, equipped with the pair-of-pants product, is an algebra over the Novikov ring $\Lambda$. This algebra is isomorphic to the (small) quantum homology $\mathrm{HQ}_{*}(M)$; see, e.g., [MS]. On the level of $\Lambda$-modules, we have

$$
\mathrm{HQ}_{*}(M)=\mathrm{H}_{*}(M) \otimes \Lambda
$$

with the tensor product grading. Thus, $\left|u \otimes e^{A}\right|=|u|+I_{c_{1}}(A)$, where $u \in \mathrm{H}_{*}(M)$ and $A \in \Gamma$. The isomorphism between $\operatorname{HF}_{*}(H)$ and $\operatorname{HQ}_{*}(M)$ is defined via the PSS-homomorphism; see [PSS] or [MS, U2]. Alternatively, it can be obtained from a homotopy of $H$ to an autonomous $C^{2}$-small Hamiltonian (under slightly more restrictive conditions than weak monotonicity, $[\mathbf{H S}]$ ) or with a somewhat different definition of the total Floer homology (as the limit of $\operatorname{HF}_{*}^{(a, b)}(H)$ as $a \rightarrow-\infty$ and $b \rightarrow \infty$, [On]).

The quantum product $u * v$ of two elements $\mathrm{H}_{*}(M)$ is defined as

$$
u * v=\sum_{A \in \Gamma}(u * v)_{A} e^{A},
$$

where the class $(u * v)_{A} \in \mathrm{H}_{*}(M)$ is determined by the condition that

$$
(u * v)_{A} \circ w=\mathrm{GW}_{A, 3}^{M}(u, v, w)
$$

for all $w \in \mathrm{H}_{*}(M)$. Here $\circ$ denotes the intersection number and $\mathrm{GW}_{A, 3}^{M}$ is the corresponding Gromov-Witten invariant; see [MS].

Note that $(u * v)_{0}=u \cap v$, where $\cap$ stands for the cap product and $u$ and $v$ are ordinary homology classes. Furthermore,

$$
|u * v|=|u|+|v|-2 n
$$

and

$$
\left|(u * v)_{A}\right|=|u|+|v|-2 n-I_{c_{1}}(A) .
$$


Also observe that $I_{\omega}(A)=-\omega(A)<0$ whenever $A \neq 0$ can be represented by a holomorphic curve. Thus, in (2.2), it suffices to limit the summation to the negative cone $I_{\omega}(A) \leq 0$. In particular, in our setting, we can write

$$
u * v=u \cap v+\sum_{k>0}(u * v)_{k} q^{k} .
$$

Here, $\left|(u * v)_{k}\right|=|u|+|v|-2 n \pm 2 N k$ when $\left.c_{1}(M)\right|_{\pi_{2}(M)} \neq 0$, with the positive or negative sign depending on whether $M$ is positive or negative monotone. This sum is finite.

The product $*$ extends to a $\Lambda$-linear, associative, graded-commutative product on $\mathrm{HQ}_{*}(M)$. The fundamental class $[M]$ is the unit in the algebra $\mathrm{HQ}_{*}(M)$. Thus, $q u=(q[M]) * u$, where $q \in \Lambda$ and $u \in \mathrm{H}_{*}(M)$, and $|q u|=|q|+|u|$. By the very definition of $\mathrm{HQ}_{*}(M)$, the ordinary homology $\mathrm{H}_{*}(M)$ is canonically embedded in $\mathrm{HQ}_{*}(M)$. The group of symplectomorphisms acts on the algebra $\mathrm{HQ}_{*}(M)$ via its action on $\mathrm{H}_{*}(M)$ and, clearly, symplectomorphisms isotopic to $i d$ act trivially.

Example 2.2. Let $M=\mathbb{C P} P^{n}$. Then $N=n+1$ and $\mathrm{HQ}_{*}\left(\mathbb{C P}^{n}\right)$ is the quotient of $\mathbb{F}[u] \otimes \Lambda$, where $u$ is the generator of $\mathrm{H}_{2 n-2}\left(\mathbb{C P}^{n}\right)$, by the ideal generated by the relation $u^{n+1}=q[M]$. Thus, $u^{k}=u \cap \cdots \cap u$ ( $k$ times) when $0 \leq k \leq n$ and $[p t] * u=q[M]$. For further examples of calculations of quantum homology and relevant references we refer the reader to, e.g., [MS].

The quantum cohomology $\mathrm{HQ}^{*}(M)$ is defined by dualizing the entire construction. We have $\mathrm{HQ}^{*}(M)=\mathrm{H}^{*}(M) \otimes \Lambda$ as graded modules over the Novikov ring $\Lambda$. The product of two ordinary cohomology classes is obtained by taking the product of their Poincaré dual homology classes $u$ and $v$ and then taking the Poincaré duals of the coefficients $(u * v)_{A}$. Note that this is an exclusive feature of monotone (with $\lambda \neq 0$ ) or negative monotone manifolds that here we can take the same Novikov ring as in the case homology; see, e.g., [MS, Example 11.1.4 and Remark 11.1.16], for further details. For our purposes, essentially for purely notational reasons, it is convenient to swap the roles of $q$ and $q^{-1}$ in the identification of the Novikov ring with the ring of Laurent polynomials. Thus, in cohomology, $|q|=2 N$.

2.3. Action selectors. The theory of Hamiltonian action selectors or spectral invariants, as they are usually referred to, was developed in its present Floer-theoretic form in $[\mathbf{O h}, \mathbf{S c}]$ although the first versions of the theory go back to $[\mathbf{H Z}, \mathbf{V i 2}]$. Here we briefly recall the main results of the theory essential for our proofs, mainly following [GG1].

Let $M$ be a closed symplectic manifold and let $H$ be a Hamiltonian on $M$. It is essential to assume here that $M$ is rational - this assumption greatly simplifies the theory (cf. [U1]) and is obviously satisfied for monotone or negative monotone manifolds. 
The action selector $\mathrm{c}_{v}$ associated with a non-zero class $v \in \mathrm{HQ}_{*}(M ; \mathbb{F}) \cong$ $\mathrm{HF}_{*}(H)$ is defined as

$$
\mathrm{c}_{v}(H)=\inf \left\{a \in \mathbb{R} \backslash \mathcal{S}(H) \mid v \in \operatorname{im}\left(i^{a}\right)\right\}=\inf \left\{a \in \mathbb{R} \backslash \mathcal{S}(H) \mid j^{a}(v)=0\right\},
$$

where $i^{a}: \mathrm{HF}_{*}^{(-\infty, a)}(H) \rightarrow \mathrm{HF}_{*}(H)$ and $j^{a}: \operatorname{HF}_{*}(H) \rightarrow \mathrm{HF}_{*}^{(a, \infty)}(H)$ are the natural "inclusion" and "quotient" maps. Then $\mathrm{c}_{v}(H)>-\infty$ as is easy to see; $[\mathbf{O h}]$.

The action selector $\mathrm{c}_{v}$ has the following properties:

(AS1) Normalization: $\mathrm{c}_{[M]}(H)=\max H$ if $H$ is autonomous and $C^{2}$-small.

(AS2) Continuity: $c_{v}$ is Lipschitz in $H$ in the $C^{0}$-topology.

(AS3) Monotonicity: $\mathrm{c}_{v}(H) \geq \mathrm{c}_{v}(K)$ whenever $H \geq K$ pointwise.

(AS4) Hamiltonian shift: $\mathrm{c}_{v}(H+a(t))=\mathrm{c}_{v}(H)+\int_{0}^{1} a(t) d t$, where $a: S^{1} \rightarrow \mathbb{R}$.

(AS5) Symplectic invariance: $\mathrm{c}_{v}(H)=\mathrm{c}_{\varphi_{*}^{-1}(v)}\left(\varphi^{*} H\right)$ for any symplectomorphism $\varphi$.

(AS6) Homotopy invariance: $\mathrm{c}_{v}(H)=\mathrm{c}_{v}(K)$ when $\varphi_{\mathrm{H}}=\varphi_{K}$ in the universal covering of the group of Hamiltonian diffeomorphisms and both $H$ and $K$ are normalized to have zero mean.

(AS7) Triangle inequality or sub-additivity: $\mathrm{c}_{v * u}(H \sharp K) \leq \mathrm{c}_{v}(H)+\mathrm{c}_{u}(K)$.

(AS8) Spectrality: $\mathrm{c}_{v}(H) \in \mathcal{S}(H)$. More specifically, there exists a capped one-periodic orbit $\bar{x}$ of $H$ such that $\mathrm{c}_{v}(H)=\mathcal{A}_{\mathrm{H}}(\bar{x})$.

(AS9) Ljusternik-Schnirelman inequality: $\mathrm{c}_{v * u}(H)<\mathrm{c}_{v}(H)$, whenever oneperiodic orbits of $H$ are isolated and $u \in \mathrm{HQ}_{*<2 n}(M)$.

This list of the properties of $\mathrm{c}$ is far from exhaustive, but it is more than sufficient for our purposes. It is worth emphasizing that the rationality assumption plays an important role in the proofs of the homotopy invariance and spectrality; see $[\mathbf{O h}, \mathbf{S c}]$ and also $[\mathbf{E P}]$ for a simple proof. (The latter property also holds in general for non-degenerate Hamiltonians. This is a non-trivial result; [U1].) The Ljusternik-Schnirelman inequality, (AS9), is established in [GG1, Proposition 6.2]. Finally note that for the triangle inequality to hold one has to work with a suitable definition of the pair-ofpants product in Floer homology; cf. [AS, U2]. We refer the reader to [U2] for a very detailed treatment of action selectors.

2.4. Carrier of the action selector. When $H$ is non-degenerate, the action selector $\mathrm{c}_{v}$ can also be evaluated as

$$
\mathrm{c}_{v}(H)=\inf _{[\sigma]=v} \mathcal{A}_{\mathrm{H}}(\sigma)
$$

where we set

$$
\mathcal{A}_{\mathrm{H}}(\sigma)=\max \left\{\mathcal{A}_{\mathrm{H}}(\bar{x}) \mid \sigma_{\bar{x}} \neq 0\right\} \text { for } \sigma=\sum \sigma_{\bar{x}} \bar{x} \in \mathrm{CF}_{|v|}(H) .
$$

The infimum here is obviously attained when $M$ is rational. Hence, there exists a cycle $\sigma=\sum \sigma_{\bar{x}} \bar{x} \in \mathrm{CF}_{|v|}(H)$, representing $v$, such that 
$\mathrm{c}_{v}(H)=\mathcal{A}_{\mathrm{H}}(\bar{x})$ for an orbit $\bar{x}$ entering $\sigma$. In other words, $\bar{x}$ maximizes the action on $\sigma$ and the cycle $\sigma$ minimizes the action over all cycles in the homology class $v$. We call such an orbit $\bar{x}$ a carrier of the action selector. Note that this is a stronger requirement than just the equality $\mathrm{c}_{v}(H)=\mathcal{A}_{\mathrm{H}}(\bar{x})$. A carrier is not in general unique, but it becomes unique when all one-periodic orbits of $H$ have distinct action values.

Our next goal is to recall a generalization of this definition, following [GG3], to the case where one-periodic orbits of $H$ are isolated but possibly degenerate. Under a $C^{2}$-small, non-degenerate perturbation $\tilde{H}$ of $H$, every such orbit $x$ splits into several non-degenerate orbits that are close to $x$. Furthermore, a capping of $x$ naturally gives rise to a capping of each of these orbits.

Definition 2.3. A capped one-periodic orbit $\bar{x}$ of $H$ is a carrier of the action selector $\mathrm{c}_{v}$ for $H$ if there exists a sequence of $C^{2}$-small, non-degenerate perturbations $\tilde{H}_{i} \stackrel{C^{2}}{\rightarrow} H$ such that one of the capped orbits which $\bar{x}$ splits into is a carrier for $\tilde{H}_{i}$. An orbit (without capping) is said to be a carrier if it turns into one for a suitable choice of capping.

It is easy to see that a carrier necessarily exists, provided that $M$ is rational and all one-periodic orbits of $H$ are isolated. As in the non-degenerate case, a carrier is of course not unique in general - different choices of sequences $\tilde{H}_{i}$ and different choices of a carrier for $\tilde{H}_{i}$ can lead to different carriers. However, it becomes unique when all one-periodic orbits of $H$ have distinct action values. In other words, under the latter requirement, the carrier is independent of the choice of the sequence $\tilde{H}_{i}$ and the choice of the carrier for $\tilde{H}_{i}$.

Picking a carrier for every $v \in \mathrm{HQ}_{*}(M)$, we obtain a map, also referred to as a carrier,

$$
\bar{\Phi}_{\mathrm{H}}: \mathrm{HQ}_{*}(M) \backslash\{0\} \rightarrow \overline{\mathcal{P}}
$$

sending $v$ to the carrier for $\mathrm{c}_{v}$. This map, of course, is not unique unless $H$ has distinct action values.

Recall that $M$ is assumed to be monotone with $\lambda \neq 0$ or negative monotone and $\left.c_{1}(M)\right|_{\pi_{2}(M)} \neq 0$. In particular, $\Gamma \cong \mathbb{Z}$ and $|q|<0$.

Clearly, when $H$ has distinct action values, $\bar{\Phi}$ is automatically equivariant with respect to recapping:

$$
\bar{\Phi}_{\mathrm{H}}(q v)=\bar{\Phi}_{\mathrm{H}}(v) \# A, \text { where } q=e^{A} .
$$

We claim that there is always a recapping-equivariant carrier $\bar{\Phi}$, i.e., a carrier satisfying (2.4). Indeed, we can pick $\bar{\Phi}$ on $\mathrm{HQ}_{d}(M)$ for all $d$ in any degree range of length $2 N$ (for instance, $[0,2 N-1]$ ) and then extend it to the entire quantum homology "by periodicity", i.e., by imposing (2.4) on $\bar{\Phi}$. 
A carrier gives rise to a map, also referred to as a carrier,

$$
\Phi: \mathrm{HQ}_{*}(M) \backslash\{0\} \rightarrow \mathcal{P}
$$

forgetting the capping. Clearly, $\Phi$ is recapping-invariant, i.e., $\Phi_{\mathrm{H}}(q v)=$ $\Phi_{\mathrm{H}}(v)$, when $\bar{\Phi}$ is recapping-equivariant.

Remark 2.4. Note that, as an immediate consequence of the definition of the carrier and continuity of the action and the mean index, we have

$$
\mathrm{c}_{v}(H)=\mathcal{A}_{\mathrm{H}}(\bar{x}) \text { and }|v|-2 n \leq \Delta_{\mathrm{H}}(\bar{x}) \leq|v|,
$$

where $\bar{x}=\bar{\Phi}(v)$, and the inequalities are strict when $x$ is weakly nondegenerate. Furthermore, the local Floer homology of $H$ at $\bar{x}$ in degree $|v|$ is non-trivial: $\operatorname{HF}_{|v|}(H, \bar{x}) \neq 0$. (This fact is established in [GG3] for $v=[M]$; but the argument applies to other homology classes word-for-word. We refer the reader to, e.g., [GG1, GG2] for a detailed discussion of the local Floer homology.) Finally note that under our requirements on $M$ it is not hard to show that $\bar{\Phi}$ can be chosen so that $\bar{\Phi}_{\mathrm{H}}(\alpha v)=\bar{\Phi}_{\mathrm{H}}(v)$ for all $\alpha \neq 0$ in $\mathbb{F}$.

Remark 2.5. We finish this discussion with one minor, fairly standard, technical point; cf. [GG3]. Namely, recall that the Floer complex of a nondegenerate Hamiltonian $H$ depends not only on $H$ but also on an auxiliary structure $J$, e.g., an almost complex structure when $M$ is weakly monotone. Moreover, the complex is defined only when suitable regularity requirements are met. As a consequence, an action selector carrier is in reality assigned to the pair $(H, J)$ rather than to just a Hamiltonian $H$ in both the nondegenerate and degenerate cases. Thus, in Definition 2.3, we tacitly assumed the presence of an auxiliary structure $J$ in the background and that the regularity requirements are satisfied for the sequence of perturbations. This can be achieved by either considering regular pairs $\left(\tilde{H}_{i}, J_{i}\right)$ with $J_{i} \rightarrow J$ or even by setting $J_{i}=J$.

\section{Proof of Theorem 1.1}

Let, as in Theorem 1.1, $\varphi_{\mathrm{H}}$ be a Hamiltonian diffeomorphism with finitely many periodic orbits of a monotone symplectic manifold $M$. (Recall that we can assume that $\left\langle c_{1}(M), \pi_{2}(M)\right\rangle \neq 0$; for otherwise $\varphi_{\mathrm{H}}$ has infinitely many periodic points; see $[\mathbf{G G} \mathbf{1}, \mathbf{H e}]$ and also $[\mathbf{G i}, \mathbf{H i}]$ ). Recall also that $\mathcal{P}$ (and $\overline{\mathcal{P}})$ stand for the collection of (capped) one-periodic orbits of $\varphi_{\mathrm{H}}$.

Although the theorem is stated in terms of cohomology, we find working with homology more intuitive at this stage. When translated to homology, the cohomological product decomposition (1.1) retains the same form

$$
u_{0} * u_{1} * \cdots * u_{\ell}=q^{\nu} u_{0}
$$


where now all $u_{j}$ are in $\mathrm{HQ}_{*}(M)$, the classes $u_{1}, \ldots, u_{\ell}$ have degree less than $2 n$, and

$$
2 n(\ell-1)-\left|u_{1}\right|-\cdots-\left|u_{\ell-1}\right|<2 N .
$$

As in (1.1), we have $\nu>0$.

In Case (ii) we simply have $u^{d} \neq 0$, where $|u|<2 n$. Recall also that now, since we are using homology, $|q|=-2 N$.

3.1. Case (i). Set

$$
\begin{aligned}
& v_{0}:=u_{0}, \\
& v_{1}:=v_{0} * u_{1}, \\
& v_{2}:=v_{1} * u_{2}, \\
& \ldots \ldots \ldots \ldots \ldots \ldots \ldots \\
& v_{\ell-1}:=v_{\ell-2} * u_{\ell-1} .
\end{aligned}
$$

It is convenient to extend the sequence $v_{j}$ with $0 \leq j \leq \ell-1$ in both directions by periodicity, using (3.1) in the definition of $v_{\ell}$. Namely, we set

$$
\begin{aligned}
& v_{\ell}:=v_{\ell-1} * u_{\ell}=q^{\nu} v_{0}, \\
& v_{\ell+1}:=v_{\ell} * u_{1}=q^{\nu} v_{1}, \\
& v_{\ell+2}:=v_{\ell+1} * u_{2}=q^{\nu} v_{2}, \\
& \ldots \ldots \ldots \ldots \ldots \ldots \ldots \ldots \ldots \ldots \ldots \ldots
\end{aligned}
$$

and

where

$$
v_{-1}:=q^{-\nu} v_{\ell-1}=v_{-2} * u_{\ell-1},
$$

$$
v_{-2}:=q^{-\nu} v_{\ell-2}=v_{-3} * u_{\ell-2},
$$

etc. As a result, we have a sequence $v_{j}$ such that

$$
v_{j+\ell}=q^{\nu} v_{j}
$$

for some $\nu>0$, and

$$
v_{j+1}=v_{j} * w_{j+1},
$$

for some $w_{j} \in \mathrm{HQ}_{*<2 n}(M)$. (Here $w_{j+1}=u_{j+1}$ for $j=0, \ldots, \ell-1$ and then again the sequence $w_{j}$ extends by periodicity.) We call such a sequence $\left\{v_{j}\right\}$ a ladder and $\ell$ the length of the ladder. Let us denote the entire ladder by $L$ and its segment $\left\{v_{0}, \ldots, v_{\ell-1}\right\}$ by $V$.

It is important for what follows that in addition to the requirements (3.3) and (3.4) we also have (3.2) satisfied, i.e., in terms of the ladder,

$$
\left|v_{0}\right|>\left|v_{1}\right|>\cdots>\left|v_{\ell-1}\right|>\left|v_{0}\right|-2 N
$$

Clearly, a ladder $L=\left\{v_{j}\right\}$ is strictly ordered by the index $\left|v_{j}\right|$ (or, to be more precise, the degree), since $\left|w_{j}\right|<2 n$. (This fact is also incorporated in (3.5).) Furthermore, for any Hamiltonian $K$ with isolated fixed points, $L$ is strictly ordered by the action, i.e.,

$$
\mathrm{c}_{v_{j}}(K)>\mathrm{c}_{v_{j+1}}(K) \text { for all } j .
$$


Here the non-strict inequality follows immediately from (3.4) and the subadditivity of the action selector, (AS7), and holds for any $K$. The strict inequality requires the fixed points of $K$ to be isolated and is a consequence of the Ljusternik-Schnirelman inequality (AS9), cf. [GG1, Proposition 6.2]. It is essential that these two orderings of $L$ coincide.

We claim that there exists a sequence of prime iterations $k_{i} \rightarrow \infty$ and a sequence of recapping-equivariant action selector carriers $\bar{\Phi}_{H^{\sharp k_{i}}}$ such that all maps $\left.\Phi_{H^{\sharp k_{i}}}\right|_{V}$ (or, equivalently, $\left.\Phi_{H^{\sharp k_{i}}}\right|_{L}$ ) coincide, i.e.,

$$
\Phi_{H^{\sharp k_{1}}}\left(v_{j}\right)=\Phi_{H^{\sharp k_{2}}}\left(v_{j}\right)=\cdots=\Phi_{H^{\sharp k_{i}}}\left(v_{j}\right)=\cdots
$$

for all $j=0, \ldots, \ell-1$ and hence, by periodicity of $L$, for all $j \in \mathbb{Z}$. In other words, all carriers $\bar{\Phi}_{H^{\sharp k_{i}}}$ assume the same value, up to recapping, on each class $v_{j}$ in $L$.

Indeed, note that since $\varphi_{\mathrm{H}}$ has finitely many periodic orbits, for every sufficiently large prime $p_{i}$, every $p_{i}$-periodic point is in fact a fixed point. Thus we start with a sequence $p_{i}$ of all sufficiently large primes. The existence of the subsequence $k_{i}$ in this sequence follows immediately from the pigeonhole principle and the existence of a recapping-equivariant carrier for any Hamiltonian. Indeed, the collection of maps from $V$ to $\mathcal{P}$ is finite. Hence, there is only a finite number of possible maps $\left.\Phi_{H^{\sharp p_{i}}}\right|_{V}$.

Remark 3.1. When the ground field $\mathbb{F}$ is finite, a similar argument shows that every infinite sequence of iterations contains an infinite subsequence $k_{i}$ such that the carriers $\bar{\Phi}_{H^{\sharp k_{i}}}$ are recapping-equivariant and all $\Phi_{H^{\sharp k_{i}}}$ are identically equal to each other on $\mathrm{HQ}_{*}(M ; \mathbb{F})$. (It is not clear however whether this would also be true when, for instance, $\mathbb{F}=\mathbb{Q}$.) In fact, for any ground field $\mathbb{F}$, the argument applies to any finite collection of non-zero elements in $\mathrm{HQ}_{*}(M ; \mathbb{F})$ in place of $V$.

Before we continue the proof, let us introduce some notation. Namely, set

$$
\bar{\Phi}_{i}=\left.\bar{\Phi}_{H^{\sharp k_{i}}}\right|_{L}: L \rightarrow \overline{\mathcal{P}} \text {. }
$$

These maps are one-to-one by (3.6). Also note that the maps $\Phi_{i}=\left.\Phi_{H^{\sharp} k_{i}}\right|_{L}$ agree, due to (3.7), and we denote them by $\Phi$ in what follows.

Next we claim that all fixed points in the image $\Phi(L)=\Phi(V)$ have the same augmented action. Thus let us pick two points $x$ and $y$ in the image. Our goal is to show that

$$
\tilde{\mathcal{A}}_{H}(x)=\tilde{\mathcal{A}}_{\mathrm{H}}(y)
$$

or, equivalently, once arbitrary cappings of $x$ and $y$ are fixed, that

$$
\mathcal{A}_{H}(\bar{x})-\mathcal{A}_{\mathrm{H}}(\bar{y})=\frac{\lambda}{2}\left(\Delta_{H}(\bar{x})-\Delta_{\mathrm{H}}(\bar{y})\right),
$$

where $\lambda$ is the monotonicity constant. (Recall that $\lambda$ and the rationality constant $\lambda_{0}$ are related by $\lambda=\lambda_{0} / N$.) 
Remark 3.2. It is essential for the proof of Case (ii) that the reasoning establishing (3.8) only uses the periodicity property (3.3) and the action ordering property (3.6). Other than relying on these two results, it is independent of the fact that $L$ is a ladder: the product requirement (3.4) does not enter the argument directly, but only via the proof of (3.6). Furthermore, to prove in the non-degenerate case that all orbits in $\Phi(V)$ are distinct we will only use (3.5).

Consider the capped orbits $\bar{x}^{k_{i}}$ and $\bar{y}^{k_{i}}$. These orbits need not be in the image of $\bar{\Phi}_{i}$ unless $\nu=1$. We denote the orbits in the image, closest to $\bar{x}^{k_{i}}$ and $\bar{y}^{k_{i}}$, by $\bar{x}_{i}$ and $\bar{y}_{i}$, respectively. In other words, $\bar{x}_{i}=q^{l} \bar{x}_{k_{i}}$ and $\bar{y}_{i}=q^{m} \bar{y}_{k_{i}}$, where the integers $l$ and $m$ are chosen so that $\bar{x}_{i}$ and $\bar{y}_{i}$ are $\operatorname{in} \operatorname{Im}\left(\bar{\Phi}_{i}\right)$ and $|l|$ and $|m|$ are as small as possible. (If the closest orbit is not unique - there can be two - we pick it in an arbitrary way. When $\nu=1$, we have $\bar{x}_{i}=\bar{x}^{k_{i}}$ and $\bar{y}_{i}=\bar{y}^{k_{i}}$.) It follows from the $q^{\nu}$-periodicity of $L$ that $|l|$ and $|m|$ do not exceed $\nu / 2$ and hence

$$
\left|\mathcal{A}_{H^{\sharp k_{i}}}\left(\bar{x}_{i}\right)-\mathcal{A}_{H^{\sharp k_{i}}}\left(\bar{x}^{k_{i}}\right)\right| \leq \frac{\lambda_{0} \nu}{2} \text { and }\left|\mathcal{A}_{H^{\sharp k_{i}}}\left(\bar{y}_{i}\right)-\mathcal{A}_{H^{\sharp k_{i}}}\left(\bar{y}^{k_{i}}\right)\right| \leq \frac{\lambda_{0} \nu}{2}
$$

and

$$
\left|\Delta_{H^{\sharp k_{i}}}\left(\bar{x}_{i}\right)-\Delta_{H^{\sharp k_{i}}}\left(\bar{x}^{k_{i}}\right)\right| \leq N \nu \text { and }\left|\Delta_{H^{\sharp k_{i}}}\left(\bar{y}_{i}\right)-\Delta_{H^{\sharp k_{i}}}\left(\bar{y}^{k_{i}}\right)\right| \leq N \nu \text {. }
$$

Indeed, among all cappings of $x$ or $y$ those coming from $L$ via $\bar{\Phi}_{i}$ for any $i$ occur periodically at least once within any interval of $\nu$ cappings.

Let us estimate the number $m_{i}$ of capped orbits in $\bar{\Phi}_{i}(L)$ between $\bar{x}_{i}$ and $\bar{y}_{i}$ using the action and index orderings. Note that the map $\bar{\Phi}_{i}$ and even its image (unless $\nu=1$ ) depend on $i$ and so does $m_{i}$. However, by (3.5) and (3.6), the two orderings agree for every $i$, and the results must be the same whether we use the index or action ordering. Without loss of generality, we may assume that $\mathcal{A}_{H}(\bar{x})>\mathcal{A}_{H}(\bar{y})$ and hence $\mathcal{A}_{H^{\sharp k_{i}}}\left(\bar{x}^{k_{i}}\right)>\mathcal{A}_{H^{\sharp k_{i}}}\left(\bar{y}^{k_{i}}\right)$.

Every class in $V$ contributes an orbit occurring periodically in $L$. From the action perspective, the period is $\lambda_{0} \nu$ by (3.1). Thus, we have

$$
\begin{aligned}
m_{i} & =\frac{|\Phi(V)|}{\lambda_{0} \nu}\left(\mathcal{A}_{H^{\sharp k_{i}}}\left(\bar{x}_{i}\right)-\mathcal{A}_{H^{\sharp k_{i}}}\left(\bar{y}_{i}\right)\right)+O(1) \\
& =\frac{|\Phi(V)|}{\lambda_{0} \nu}\left(\mathcal{A}_{H^{\sharp k_{i}}}\left(\bar{x}^{k_{i}}\right)-\mathcal{A}_{H^{\sharp k_{i}}}\left(\bar{y}^{k_{i}}\right)\right)+O(1),
\end{aligned}
$$

where we use (3.9) to pass to the second equality. Henceforth $O(1)$ stands for a term bounded from above and below by a constant independent of $i$. 
From the index perspective, the period is $2 N \nu$ and we have, using now $(3.10)$,

$$
\begin{aligned}
m_{i} & =\frac{|\Phi(V)|}{2 N \nu}\left(\Delta_{H^{\sharp k_{i}}}\left(\bar{x}_{i}\right)-\Delta_{H^{\sharp k_{i}}}\left(\bar{y}_{i}\right)\right)+O(1) \\
& =\frac{|\Phi(V)|}{2 N \nu}\left(\Delta_{H^{\sharp k_{i}}}\left(\bar{x}^{k_{i}}\right)-\Delta_{H^{\sharp k_{i}}}\left(\bar{y}^{k_{i}}\right)\right)+O(1) .
\end{aligned}
$$

Equating the results, we see after a simple algebraic manipulation that

$$
\frac{1}{\lambda_{0}}\left(\mathcal{A}_{H^{\sharp k_{i}}}\left(\bar{x}^{k_{i}}\right)-\mathcal{A}_{H^{\sharp k_{i}}}\left(\bar{y}^{k_{i}}\right)\right)=\frac{1}{2 N}\left(\Delta_{H^{\sharp k_{i}}}\left(\bar{x}^{k_{i}}\right)-\Delta_{H^{\sharp k_{i}}}\left(\bar{y}^{k_{i}}\right)\right)+O(1) .
$$

Dividing by $k_{i}$ and passing to the limit as $k_{i} \rightarrow \infty$, we arrive at (3.8) since the action and the mean index are homogeneous with respect to the iteration.

Finally set

$$
x_{j}=\Phi\left(v_{j}\right), \text { for } j=0, \ldots, \ell-1 .
$$

To finish the proof, we only need to show that these orbits are distinct, i.e., $|\Phi(V)|=\ell$. Let us cap the orbits using say $\bar{\Phi}_{1}$, i.e., by setting $\bar{x}_{j}=\bar{\Phi}_{1}\left(v_{j}\right)$. Then by (3.6), we have

$$
\mathcal{A}_{H^{\sharp k_{1}}}\left(\bar{x}_{0}\right)>\mathcal{A}_{H^{\sharp} k_{1}}\left(\bar{x}_{1}\right)>\cdots>\mathcal{A}_{H^{\sharp} k_{1}}\left(\bar{x}_{\ell-1}\right)>\mathcal{A}_{H^{\sharp k_{1}}}\left(\bar{x}_{0}\right)-\lambda_{0} \nu .
$$

When $\nu=1$, this immediately implies that no two orbits $\Phi(V)$ are recappings of each other. When $\nu>1$, we use the Conley-Zehnder index - hence the non-degeneracy assumption - rather than the action to distinguish the orbits. Namely, recall that $\mu_{\mathrm{CZ}}\left(\bar{x}_{j}\right)=\left|v_{j}\right|-n$. Thus, (3.5) is equivalent to

$$
\mu_{\mathrm{CZ}}\left(\bar{x}_{0}\right)>\mu_{\mathrm{CZ}}\left(\bar{x}_{1}\right)>\cdots>\mu_{\mathrm{CZ}}\left(\bar{x}_{\ell-1}\right)>\mu_{\mathrm{CZ}}\left(\bar{x}_{0}\right)-2 N,
$$

and, as a consequence, $\left|\mu_{\mathrm{CZ}}\left(\bar{x}_{j}\right)-\mu_{\mathrm{CZ}}\left(\bar{x}_{k}\right)\right|<2 N$. Hence, all orbits in $\Phi(V)$ are distinct.

3.2. Case (ii). The idea of the proof is that for the previous argument to go through we do not need the product decomposition (3.1) to hold literally. It is in fact sufficient to have action selector carriers taking the same value (up to a capping) on the left and right hand sides of (3.1) for the sequence of iterations $k_{i}$. The proof shares many common elements with the reasoning in Case (i) and below we only detail the necessary changes.

Consider the finite collection

$$
U=\left\{u, u^{2}, \ldots, u^{d}\right\}
$$

of non-zero elements in $\mathrm{HQ}_{*<2 n}(M)$, where

$$
\left|u^{r}\right|=2 n-(2 n-|u|) r .
$$

By arguing exactly as in the proof of Case (i), it is easy to find a sequence of prime iterations $k_{i}$ and a sequence of recapping-equivariant action selector carriers $\bar{\Phi}_{H^{\sharp k_{i}}}$ such that the maps $\Phi_{H^{\sharp k_{i}}}$ agree on $U$; cf. Remark 3.1. Let us denote the resulting map $U \rightarrow \mathcal{P}$ by $\Phi$. 
Since $|U|=d$ is sufficiently large, e.g.,

$$
|U|=\left\lceil\frac{2 N|\mathcal{P}|}{2 n-|u|}\right\rceil+1
$$

there exist $s_{-}$and $s_{+}$, both in the range $[1, d]$, such that $s_{+}>s_{-}+2 N /(2 n-$ $|u|)$ or, equivalently,

$$
\left|u^{s_{-}}\right|-\left|u^{s+}\right|>2 N
$$

and

$$
\Phi\left(u^{s_{-}}\right)=\Phi\left(u^{s_{+}}\right) .
$$

This is again an immediate consequence of the pigeonhole principle. Indeed, otherwise the number of classes mapped to any point in $\mathcal{P}$ would not exceed $2 N /(2 n-|u|)$, and hence we would have $|U| \leq 2 N|\mathcal{P}| /(2 n-|u|)$.

Let, as in Section 2.2, $A$ be the generator of $\Gamma$ such that $I_{c_{1}}(A)=-2 N$, i.e., $q=e^{A}$. For every $k_{i}$,

$$
\bar{\Phi}_{H^{\sharp k_{i}}}\left(u^{s_{+}}\right)=\bar{\Phi}_{H^{\sharp k_{i}}}\left(u^{s_{-}}\right) \#(\nu A),
$$

where $\nu=\left(s_{+}-s_{-}\right)(2 n-|u|) / 2 N$ since, due to the non-degeneracy assumption,

$$
\mu_{\mathrm{CZ}}\left(\bar{\Phi}_{H^{\sharp k_{i}}}\left(u^{s_{+}}\right)\right)=\left|u^{s_{+}}\right|-n \quad \text { and } \quad \mu_{\mathrm{CZ}}\left(\bar{\Phi}_{H^{\sharp k_{i}}}\left(u^{s_{-}}\right)\right)=\left|u^{s_{-}}\right|-n .
$$

In particular, $\nu$ is independent of $k_{i}$. (One can bypass this reference to nondegeneracy by observing that there are only finitely many possible values of $\nu$, as a simple mean index argument shows, and then by passing to a subsequence of iterations.)

Let us set $\ell=\lfloor 2 N /(2 n-|u|)\rfloor$. Consider the finite sequence $V$ formed by $\ell$ classes

$$
\begin{aligned}
& v_{0}:=u^{s_{-}}, \\
& v_{1}:=u^{s_{-}+1}=v_{0} * u, \\
& \ldots \ldots \ldots \ldots \ldots \ldots \ldots \ldots \ldots \ldots \ldots \ldots \ldots \\
& v_{\ell-1}:=u^{s_{-}+\ell-1}=v_{\ell-2} * u,
\end{aligned}
$$

which we extend in both directions by $q^{\nu}$-periodicity to have (3.3) satisfied. In other words, we set

$$
v_{\ell}=q^{\nu} v_{0}, \quad v_{\ell+1}=q^{\nu} v_{1}, \quad \ldots
$$

and

$$
v_{-1}=q^{-\nu} v_{\ell-1}, \quad v_{-2}=q^{-\nu} v_{\ell-2}, \quad \ldots
$$

Note that

$$
u^{s_{+}}=v_{\ell-1} * u^{s_{+}-s_{-}-\ell+1}, \quad \text { where } s_{+}-s_{-}-\ell+1>0
$$

due to our choice of $\ell$. Furthermore, for all $i$, we have

$$
\mathrm{c}_{u^{s_{+}}}\left(H^{\sharp k_{i}}\right)=\mathrm{c}_{v_{\ell}}\left(H^{\sharp k_{i}}\right)
$$

by (3.12) and the definition of $v_{\ell}$. 
The sequence $L=\left\{v_{j}\right\}$ is not a ladder. For (3.4) need not hold for $j=\ell$. However, we claim that $L$ is still strictly ordered by the index and the action (for the Hamiltonians $H^{\sharp k_{i}}$ ) and that the two orderings agree.

For the index ordering, this immediately follows from (3.11) and our choice of $\ell$. To see that $L$ is strictly ordered by the action, i.e., (3.6) holds for $K=H^{\sharp k_{i}}$, we argue as follows. For $j$ in the range from 0 to $\ell-2$, exactly as in Case (i) this follows from (AS9) for any Hamiltonian with isolated fixed points. For $j=\ell-1$, we have

$$
\begin{aligned}
\mathrm{c}_{v_{\ell}}\left(H^{\sharp k_{i}}\right) & =\mathrm{c}_{v_{0}}\left(H^{\sharp k_{i}}\right)-\nu \lambda_{0} \\
& =\mathrm{c}_{u^{s_{-}}}\left(H^{\sharp k_{i}}\right)-\nu \lambda_{0} \\
& =\mathrm{c}_{u^{s_{+}}}\left(H^{\sharp k_{i}}\right) \\
& <\mathrm{c}_{v_{\ell-1}}\left(H^{\sharp k_{i}}\right) .
\end{aligned}
$$

Here the third equality follows from (3.12). The ultimate inequality is again a consequence of the Ljusternik-Schnirelman inequality, (AS9), and (3.13) and (3.14). Hence, the required inequalities (3.6) hold for $j=0, \ldots, \ell-1$ and, by periodicity, for all $j$ and $K=H^{\sharp k_{i}}$.

Finally note that (3.5) is satisfied by the definition of $L$ and $\ell$. With these observations in mind, the proof is finished exactly in the same way in Case (i); cf. Remark 3.2.

\section{Proof of Theorem 1.8}

The proof follows roughly the same line of reasoning as the argument in [GG3] with some changes in the general logic of the proof. Throughout this section, we will use the notation and conventions from Sections 2 and 3.

Arguing by contradiction, assume that all periodic orbits of $\varphi_{\mathrm{H}}$ with sufficiently large period are iterated. In particular, for every large prime $k$ the set of contractible $k$-periodic orbits is naturally identified with the set $\mathcal{P}$ of contractible one-periodic orbits. Then there exists a sequence of primes $k_{i} \rightarrow \infty$ and a sequence of recapping-equivariant action carriers $\bar{\Phi}_{H^{\sharp k_{i}}}$ such that

$$
\Phi_{H^{\sharp k_{1}}}([M])=\Phi_{H^{\sharp k_{2}}}([M])=\cdots .
$$

Indeed, for a large prime $k$, the carrier $\Phi_{H^{\sharp k}}$ takes values in $\mathcal{P}$ and the assertion follows from the pigeonhole principle. We denote the resulting fixed point by $x$. Thus, we have

$$
\Phi_{H^{\sharp k_{i}}}([M])=x^{k_{i}} .
$$

Set

$$
\bar{\Phi}_{H^{\sharp k_{i}}}([M])=\bar{x}_{k_{i}} .
$$


Let us first focus on the capped orbit $\bar{x}_{k_{1}}$. By Remark 2.4 and, in particular, (2.5), we have

$$
0 \leq \Delta_{H^{\sharp k_{1}}}\left(\bar{x}_{k_{1}}\right) \leq 2 n \quad \text { and } \quad \mathcal{A}_{H^{\sharp k_{1}}}\left(\bar{x}_{k_{1}}\right)=\mathrm{c}_{[M]}\left(H^{\sharp k_{1}}\right) .
$$

We treat the cases where the mean index is zero and where it is positive separately. These are the so-called degenerate and weakly non-degenerate cases of the Conley conjecture.

The degenerate case: $\boldsymbol{\Delta}_{\boldsymbol{H}^{\sharp k_{1}}}\left(\overline{\boldsymbol{x}}_{\boldsymbol{k}_{1}}\right)=\mathbf{0}$. Note that we can, without loss of generality, take $k_{1}$ arbitrarily large. More specifically, we can assume that $k_{1}$ is so large that none of the Floquet multipliers, different from one, of any one-periodic orbit $y \in \mathcal{P}$ is a $k_{1}$ th root of unity. In other words, using the terminology from [GG2], $k_{1}$ is an admissible iteration of $\varphi_{\mathrm{H}}$. Furthermore, we require $k_{1}$ to be large enough to ensure that $\Delta_{\mathrm{H}}(y)=0$ $\bmod 2 N$ whenever $k_{1} \Delta_{\mathrm{H}}(y)=0 \bmod 2 N$ for any $y \in \mathcal{P}$. (Here we treat the mean index as an element of $\mathbb{R} / 2 N \mathbb{Z}$, which is obviously well-defined, i.e., independent of the capping.)

In particular, we have

$$
k_{1} \Delta_{\mathrm{H}}(x)=\Delta_{H^{\sharp k_{1}}}\left(x^{k_{1}}\right)=\Delta_{H^{\sharp k_{1}}}\left(\bar{x}_{k_{1}}\right)=0 \bmod 2 N,
$$

and hence $\Delta_{\mathrm{H}}(x)=0 \bmod 2 N$. As a consequence, $\Delta_{\mathrm{H}}(\bar{x})=0$ for a suitable capping $\bar{x}$ of $x$. With this capping, $\Delta_{H^{\sharp k_{1}}}\left(\bar{x}^{k_{1}}\right)=\Delta_{H^{\sharp k_{1}}}\left(\bar{x}_{k_{1}}\right)$ in $\mathbb{Z}$, and therefore $\bar{x}^{k_{1}}=\bar{x}_{k_{1}}$, since $M$ is negative monotone.

We claim that $\bar{x}$ is a so-called symplectically degenerate maximum of $H$, i.e., $\operatorname{HF}_{2 n}(H, \bar{x}) \neq 0$ and $\Delta_{\mathrm{H}}(\bar{x})=0$; [GG1]. (See also [Gi, GG2, He] for the definition, a detailed discussion and applications of this notion, which originates from Hingston's proof of the Conley conjecture for tori; see [Hi].) The vanishing of the mean index has already been established. On the other hand, the local Floer homology of $\bar{x}$ in degree $2 n$ does not vanish since

$$
\mathrm{HF}_{2 n}(H, \bar{x})=\mathrm{HF}_{2 n}\left(H^{\sharp k_{1}}, \bar{x}^{k_{1}}\right) \neq 0 .
$$

Here, the first equality is a consequence of the persistence of local Floer homology for admissible iterations established in [GG2] and the second one follows from Remark 2.4.

In the presence of a symplectically degenerate maximum, the Conley conjecture (the existence of simple orbits with arbitrarily large period) is proved for rational, and in particular negative-monotone, symplectic manifolds in [GG1]. This concludes the proof of Theorem 1.8 in the degenerate case.

The weakly non-degenerate case: $\boldsymbol{\Delta}_{\boldsymbol{H}^{\sharp k_{1}}}\left(\overline{\boldsymbol{x}}_{\boldsymbol{k}_{\mathbf{1}}}\right)>\boldsymbol{0}$. Set $l_{i}=\left\lfloor k_{i} / k_{1}\right\rfloor$ and let $r_{i}$ to be the remainder of dividing $k_{i}$ by $k_{1}$, i.e., $l_{i} k_{1}+r_{i}=k_{i}$ and 
$0 \leq r_{i}<k_{1}$. Define $\nu_{i}$ by

$$
\bar{x}_{k_{i}}=\bar{x}_{k_{1}}^{l_{i}} \#\left(\nu_{i} A\right)
$$

where $A$ is a generator of $\Gamma$.

Looking at the action values on $\bar{x}_{k_{i}}$, we have,

$$
\begin{aligned}
\mathcal{A}_{H^{\sharp k_{i}}}\left(\bar{x}_{k_{i}}\right) & =\mathrm{c}_{[M]}\left(H^{\sharp k_{i}}\right) \\
& \leq l_{i} \mathrm{c}_{[M]}\left(H^{\sharp k_{1}}\right)+\mathrm{c}_{[M]}\left(H^{\sharp r_{i}}\right) \\
& \leq l_{i} \mathrm{c}_{[M]}\left(H^{\sharp k_{1}}\right)+\mathrm{const},
\end{aligned}
$$

where const stands for a constant independent of $i$. Here, the first inequality follows from the sub-additivity of the action selector.

On the other hand, by (4.1) and since the action is homogeneous,

$$
\mathcal{A}_{H^{\sharp k_{i}}}\left(\bar{x}_{k_{i}}\right)=l_{i} \mathcal{A}_{H^{\sharp k_{1}}}\left(\bar{x}_{k_{1}}\right)+\nu_{i} I_{\omega}(A),
$$

and thus

$$
\nu_{i} I_{\omega}(A) \leq \text { const. }
$$

Examining the mean indexes, we obtain in a similar vein that

$$
\Delta_{H^{\sharp k_{i}}}\left(\bar{x}_{k_{i}}\right)=l_{i} \Delta_{H^{\sharp k_{1}}}\left(\bar{x}_{k_{1}}\right)+\nu_{i} I_{c_{1}}(A),
$$

where we used again (4.1) and the homogeneity of the mean index. By the weak non-degeneracy assumption we have $\Delta_{H^{\sharp k_{1}}}\left(\bar{x}_{k_{1}}\right)>0$, and we conclude that

$$
\nu_{i} I_{c_{1}}(A) \rightarrow-\infty
$$

as $k_{i} \rightarrow \infty$. Therefore, since $M$ is negative monotone,

$$
\nu_{i} I_{\omega}(A) \rightarrow \infty
$$

which is impossible due to (4.2). This contradiction completes the proof of the theorem.

\section{References}

[AS] A. Abbondandolo and M. Schwarz, Floer homology of cotangent bundles and the loop product, Geom. Top., 14 (2010), 1569-1722.

[AK] D.V. Anosov and A.B. Katok, New examples in smooth ergodic theory. Ergodic diffeomorphisms (in Russian), Trudy Moskov. Mat. Obšč., 23(1970), 3-36.

[Be] A. Bertram, Quantum Schubert calculus, Adv. Math., 128 (1997), 289-305.

[Ek] I. Ekeland, Une théorie de Morse pour les systèmes hamiltoniens convexes, Ann. Inst. H. Poincaré Anal. Non Linéaire, 1 (1984), 19-78.

[EH] I. Ekeland and H. Hofer, Convex Hamiltonian energy surfaces and their periodic trajectories, Comm. Math. Phys., 113 (1987), 419-469. 
[EP] M. Entov and L. Polterovich, Rigid subsets of symplectic manifolds, Compos. Math., 145 (2009), 773-826.

[Es] J. Espina, On the mean Euler characteristic of contact manifold, 2010, arXiv:1011.4364.

[FH] A. Fathi and M.R. Herman, Existence de difféomorphismes minimaux. dynamical systems, Vol. I - Warsaw, Astérisque, 49 (1977), 37-59.

[FK] B. Fayad and A. Katok, Constructions in elliptic dynamics, Ergodic Theory Dynam. Syst., 24 (2004), 1477-1520.

[FO] K. Fukaya and K. Ono, Arnold conjecture and Gromov-Witten invariant, Topology, 38 (1999), 933-1048.

[FOOO] K. Fukaya, Y.-G. Oh, H. Ohta and K. Ono, Lagrangian intersection Floer theory: anomaly and obstruction, Ed. S.-T. Yau, Parts I and II, AMS/IP Studies in Advanced Mathematics, vol. 46.1 and 46.2, American Mathematical Society, Providence, RI; International Press, Somerville, MA, 2009.

[Gi] V.L. Ginzburg, The Conley conjecture, Ann. of Math., 172 (2010), 1127-1180.

[GG1] V.L. Ginzburg and B.Z. Gürel, Action and index spectra and periodic orbits in Hamiltonian dynamics, Geom. Topol., 13 (2009), 2745-2805.

[GG2] V.L. Ginzburg and B.Z. Gürel, Local Floer homology and the action gap, J. Sympl. Geom., 8 (2010), 323-357.

[GG3] V.L. Ginzburg and B.Z. Gürel, Conley conjecture for negative monotone symplectic manifolds, Int. Math. Res. Not. IMRN, 2012(8) (2011), 1748-1767, doi:10.1093/imrn/rnr081.

[GK] V.L. Ginzburg and E. Kerman, Homological resonances for Hamiltonian diffeomorphisms and Reeb flows, Int. Math. Res. Not. IMRN, 2010(1) (2010), 53-68.

[GGK] V. Guillemin, V. Ginzburg and Y. Karshon, Moment maps, Cobordisms, and Hamiltonian Group actions, Mathematical Surveys and Monographs, 98. American Mathematical Society, Providence, RI, 2002.

[He] D. Hein, The Conley conjecture for irrational symplectic manifolds, J. Sympl. Geom. 10 (2012), 183-202.

[Hi] N. Hingston, Subharmonic solutions of Hamiltonian equations on tori, Ann. Math., 170 (2009), 525-560.

[HS] H. Hofer and D. Salamon, Floer homology and Novikov rings, in Eds: H. Hofer, C.H. Taubes, A. Weinstein, E. Zehnder, 'The Floer memorial volume', 483-524, Progr. Math., 133, Birkhäuser, Basel, 1995.

[HWZ1] H. Hofer, K. Wysocki and E. Zehnder, SC-smoothness, retractions and new models for smooth spaces, Discrete Contin. Dyn. Syst., 28 (2010), 665-788.

[HWZ2] H. Hofer, K. Wysocki and E. Zehnder, Applications of polyfold theory I: the polyfolds of Gromov-Witten theory, 2011, arXiv:1107.2097.

[HZ] H. Hofer and E. Zehnder, Symplectic invariants and Hamiltonian dynamics, Birkäuser, 1994.

[Ka] R. Kaufmann, The intersection form in $H_{*}\left(\bar{M}_{0, n}\right)$ and the explicit Künneth formula in quantum cohomology, Int. Math. Res. Not., 19 (1996), 929-952.

[LM] H.B. Lawson and M.-L. Michelsohn, Spin geometry, Princeton Mathematical Series, 38, Princeton University Press, Princeton, NJ, 1989.

[LT] G. Liu and G. Tian, Floer homology and Arnold conjecture, J. Differ. Geom., 49 (1998), 1-74. 
[Lo] Y. Long, Index theory for symplectic paths with applications, Progress in Mathematics, 207. Birkhäuser Verlag, Basel, 2002.

[MS] D. McDuff and D. Salamon, J-holomorphic curves and symplectic topology, Colloquium publications, vol. 52, AMS, Providence, RI, 2004.

[Oh] Y.-G. Oh, Construction of spectral invariants of Hamiltonian paths on closed symplectic manifolds, in Eds: J.E. Marsden, T.S. Ratiu, 'The breadth of symplectic and Poisson geometry', 525-570, Progr. Math., 232, Birkhäuser, Boston, MA, 2005.

[On] K. Ono, On the Arnold conjecture for weakly monotone symplectic manifolds, Invent. Math., 119 (1995), 519-537.

[PSS] S. Piunikhin, D. Salamon and M. Schwarz, Symplectic Floer-Donaldson theory and quantum cohomology, in 'Contact and symplectic geometry' (C.B. Thomas ed.), Cambridge, 1994, 171-200; Publ. Newton Inst., 8, Cambridge University Press, Cambridge, 1996.

[Po] L. Polterovich, Hamiltonian loops and Arnold's principle, in Eds: A. Khovanskii, A. Varchenko, V. Vassiliev. (Translation edited by A.B. Sossinsky), 'Topics in singularity theory', 181-187, Amer. Math. Soc. Transl. Ser. 2, 180, Amer. Math. Soc., Providence, RI, 1997.

[Sa] D.A. Salamon, Lectures on Floer homology, in Symplectic Geometry and Topology, Eds: Y. Eliashberg and L. Traynor, IAS/Park City Mathematics Series, 7 (1999), 143-230.

[SZ] D. Salamon and E. Zehnder, Morse theory for periodic solutions of Hamiltonian systems and the Maslov index, Comm. Pure Appl. Math., 45 (1992), 13031360 .

[Sc] M. Schwarz, On the action spectrum for closed symplectically aspherical manifolds, Pacific J. Math., 193 (2000), 419-461.

[ST] B. Siebert and G. Tian, On quantum cohomology rings of Fano manifolds and a formula of Vafa and Intriligator, Asian J. Math., 1 (1997), 679-695.

[Ta] H. Tamvakis, Gromov-Witten invariants and quantum cohomology of Grassmannians, in Ed: P. Pragacz, 'Topics in cohomological studies of algebraic varieties', 271-297, Trends Math., Birkhäuser, Basel, 2005.

[Vi1] C. Viterbo, Equivariant Morse theory for starshaped Hamiltonian systems, Trans. Amer. Math. Soc., 311 (1989), 621-655.

[Vi2] C. Viterbo, Symplectic topology as the geometry of generating functions, Math. Ann., 292 (1992), 685-710.

[U1] M. Usher, Spectral numbers in Floer theories, Compos. Math., 144 (2008), 1581-1592.

[U2] M. Usher, Deformed Hamiltonian Floer theory, capacity estimates, and Calabi quasimorphisms, Geom. Topol. 15 (2011), 1313-1417.

\author{
Department of Mathematics \\ VANDERBILT UNIVERSITY \\ 1326 Stevenson Center \\ NASHVILLE \\ TN 37240 \\ E-mail address: michael.j.chance@vanderbilt.edu
}


Department of Mathematics

UC SANTA CRUZ

SANTA CRUZ

CA 95064

E-mail address: ginzburg@ucsc.edu

Department of Mathematics

VANDERBILT UNIVERSITY

NASHVILLE

TN 37240

E-mail address: basak.gurel@vanderbilt.edu

Received 01/26/2012, accepted 07/06/2012

The work was partially supported by the NSF and by the faculty research funds of the University of California, Santa Cruz. The authors are grateful to Yael Karshon and Yaron Ostrover for fruitful discussions and the referee for useful remarks. 